Article

\title{
Longitudinal T2 Mapping and Texture Feature Analysis in the Detection and Monitoring of Experimental Post-Traumatic Cartilage Degeneration
}

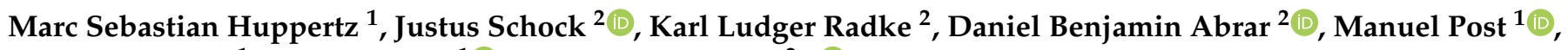 \\ Christiane Kuhl ${ }^{1}$, Daniel Truhn ${ }^{1}$ (D) and Sven Nebelung ${ }^{2, *(D)}$ \\ 1 Department of Diagnostic and Interventional Radiology, Aachen University Hospital, \\ 52074 Aachen, Germany; mhuppertz@ukaachen.de (M.S.H.); mpost@ukaachen.de (M.P.); \\ ckuhl@ukaachen.de (C.K.); dtruhn@ukaachen.de (D.T.) \\ 2 Department of Diagnostic and Interventional Radiology, Medical Faculty, University Dusseldorf, \\ 40225 Dusseldorf, Germany; justus.schock@med.uni-duesseldorf.de (J.S.); \\ ludger.radke@med.uni-duesseldorf.de (K.L.R.); DanielBenjamin.Abrar@med.uni-duesseldorf.de (D.B.A.) \\ * Correspondence: sven.nebelung@med.uni-duesseldorf.de
}

\section{check for} updates

Citation: Huppertz, M.S.; Schock, J.; Radke, K.L.; Abrar, D.B.; Post, M.; Kuhl, C.; Truhn, D.; Nebelung, S. Longitudinal T2 Mapping and Texture Feature Analysis in the Detection and Monitoring of Experimental Post-Traumatic Cartilage Degeneration. Life 2021, 11, 201. https://doi.org/10.3390/ life11030201

Academic Editor: Nicola Maffulli

Received: 30 January 2021

Accepted: 3 March 2021

Published: 5 March 2021

Publisher's Note: MDPI stays neutral with regard to jurisdictional claims in published maps and institutional affiliations.

Copyright: (C) 2021 by the authors. Licensee MDPI, Basel, Switzerland. This article is an open access article distributed under the terms and conditions of the Creative Commons Attribution (CC BY) license (https:/ / creativecommons.org/licenses/by/ $4.0 /)$.

\begin{abstract}
Background: Traumatic cartilage injuries predispose articulating joints to focal cartilage defects and, eventually, posttraumatic osteoarthritis. Current clinical-standard imaging modalities such as morphologic MRI fail to reliably detect cartilage trauma and to monitor associated posttraumatic degenerative changes with oftentimes severe prognostic implications. Quantitative MRI techniques such as T2 mapping are promising in detecting and monitoring such changes yet lack sufficient validation in controlled basic research contexts. Material and Methods: 35 macroscopically intact cartilage samples obtained from total joint replacements were exposed to standardized injurious impaction with low $(0.49 \mathrm{~J}, \mathrm{n}=14)$ or high $(0.98 \mathrm{~J}, \mathrm{n}=14)$ energy levels and imaged before and immediately, $24 \mathrm{~h}$, and $72 \mathrm{~h}$ after impaction by T2 mapping. Contrast, homogeneity, energy, and variance were quantified as features of texture on each T2 map. Unimpacted controls ( $n=7)$ and histologic assessment served as reference. Results: As a function of impaction energy and time, absolute T2 values, contrast, and variance were significantly increased, while homogeneity and energy were significantly decreased. Conclusion: T2 mapping and texture feature analysis are sensitive diagnostic means to detect and monitor traumatic impaction injuries of cartilage and associated posttraumatic degenerative changes and may be used to assess cartilage after trauma to identify "cartilage at risk".
\end{abstract}

Keywords: cartilage; focal cartilage defect; posttraumatic osteoarthritis; T2 mapping; texture analysis

\section{Introduction}

Associated with knee symptoms and dysfunction, focal cartilage lesions are common in the general population. Hjelle et al. reported (osteo)chondral lesions (of any type) in $61 \%$ of their patients undergoing knee arthroscopy [1]. These findings were confirmed by other studies, too [2-4]. The tissue's limited intrinsic healing capacity and the progressive nature of cartilage lesions warrant additional diagnostic and therapeutic efforts to prevent osteoarthritis $(\mathrm{OA})$ and its great socioeconomic and personal disease burden.

While the aetiology of focal cartilage lesions is multifactorial, focal cartilage lesions are often the result of trauma [5,6]: Meniscus and anterior cruciate ligament (ACL) injuries bring about instability and predispose the joint to cartilage lesions [5]. Similar dispositions are incurred by patellar dislocations. The prevalence rates of cartilage lesions in the patellofemoral joint are $71 \%, 82 \%$, and $97 \%$ in acute, recurrent, and chronic dislocators, respectively [6]. Other aetiologic factors are fractures, soft-tissue injuries, and repetitive microtraumatizations that result in surface incongruity, altered joint kinematics, and chronic degenerative changes, thereby predisposing to cartilage lesions, too. Consequently, post- 
traumatic OA (PTOA) accounts for nearly $12 \%$ of all cases of symptomatic OA in the United States [7].

Due to its high soft tissue contrast and spatial resolution, non-invasiveness, and lack of radiation, Magnetic Resonance Imaging (MRI) is clearly the most powerful diagnostic tool of contemporary clinical medicine and the superordinate standard imaging modality for suspected joint and cartilage disorders [8-10]. However, numerous studies have indicated the limitations of clinical-standard morphologic MRI techniques in the detection of cartilage lesions with variable sensitivities of $45 \%$ to $74 \%$ [11,12]. With the positive predictive value equally variable, morphologic MRI techniques are (i) not able to reliably indicate the presence (or absence) of cartilage lesions and (ii) particularly limited in detecting early, potentially reversible cartilage lesions. Consequently, quantitative MRI techniques such as T2 and T1 $\rho$ mapping have received ever-increasing scientific and clinical attention over the last decades $[13,14]$. These techniques quantify biophysical tissue properties on the compositional and ultrastructural level beyond mere morphology. Widely available on clinical MRI scanners and conveniently acquired with an additional scan time of 5 min, the addition of a T2 mapping sequence to a routine imaging protocol improved sensitivity in the detection of (early) cartilage lesions [15]. T2 mapping is a robust, clinically and scientifically well-validated, and commonly used technique to assess cartilage status [13,14]. Moreover, T2 mapping is closely associated with relevant structural and compositional tissue features such as collagen content, collagen network organization and integrity, and water content [16]. Consequently, a solid body of evidence has been collected that indicates the potential of T2 mapping in evaluating posttraumatic cartilage changes [17-23].

Instead of merely quantifying T2 values of the superficial and deep cartilage zones in a pixel-wise manner, recent approaches have relied on more comprehensive post hoc approaches for image analysis such as texture feature analyses. During cartilage degeneration, collagen network integrity and proteoglycan content are lost [24]. The increasing degrees of tissue disruption and disorganization translate to altered spatial distributions of T2 and may be quantified as markers of heterogeneity based on textural features. In degenerated cartilage, T2 values tend to be elevated with greater local heterogeneity [17,25] as has been demonstrated for cartilage lesions [26], symptomatic OA and patients at risk of developing OA $[25,27,28]$, and after ACL injury [29].

Despite this wealth of clinical knowledge, a basic understanding of the posttraumatic degenerative changes in cartilage and their imaging correlates is lacking. The present study's objective was to contribute to this understanding by bringing together intact human articular cartilage, standardized injurious impaction loading with variable impaction energies, and T2 mapping and post hoc texture feature analysis. To this end, (histologically referenced) intact cartilage tissue was subject to impaction loading using a drop-tower device as an established model for inducing posttraumatic degenerative changes [30-32], and imaged longitudinally to study these changes as a function of time and impaction energy, i.e., trauma severity. Our hypotheses were that (i) variable impaction energies induce variable progressive posttraumatic degenerative changes in cartilage and that (ii) these changes are reflected by the T2 maps and associated descriptive statistics and texture features.

\section{Materials and Methods}

\subsection{Cartilage Sample Preparations}

Following informed consent and Institutional Ethical Review Board approval (Ethical Committee, RWTH Aachen University, Germany, AZ EK 157/13), we obtained human articular cartilage-bone samples from 24 patients undergoing total knee replacement surgery at our institution (10 male, 14 female; mean age 63.4 years [range: 53-89 years]) [33-39]. Primary OA of the knee as determined radiographically and clinically was defined as the inclusion criterion, while all forms of secondary OA as well as previous trauma and/or surgery, and other bone and joint diseases were defined as exclusion criteria. Immediately after intraoperative excision, cartilage-bone material was collected in sterile Dulbecco's 
modified Eagle's medium (DMEM) containing $100 \mathrm{U} / \mathrm{mL}$ penicillin, $100 \mu \mathrm{g} / \mathrm{mL}$ gentamycin, and 1.25 U/mL amphotericin B (all from Gibco-BRL, Gaithersburg, MD, USA). Subsequent preparations were carried out as before $[32,37,38]$. To maintain topoanatomic consistency, only cartilage-bone material from the lateral femoral condyle was included. Samples were cut to standard size (length $\times$ width: $15 \times 15[\mathrm{~mm}]$ ) and any cancellous bone was removed while preserving the subchondral lamella and keeping the surface as plain as possible. Samples were then graded according to the Outerbridge classification that assesses the tissue's macroscopic appearance [40]. Only Outerbridge-grade 0 samples, i.e., normal cartilage without softening or swelling, were included. For reference purposes, three notches were created using a rongeur, i.e., two notches at opposing sample sides to define the mid-sagittal imaging plane and a third notch to define an orthogonal plane. The intersection of these planes was defined as the sample centre point (Figure 1a). Additionally, macroscopically similar cartilage tissue immediately adjacent to the actual cartilage sample was prepared along the mid-sagittal plane to assess baseline histologic characteristics of the cartilage-bone material.

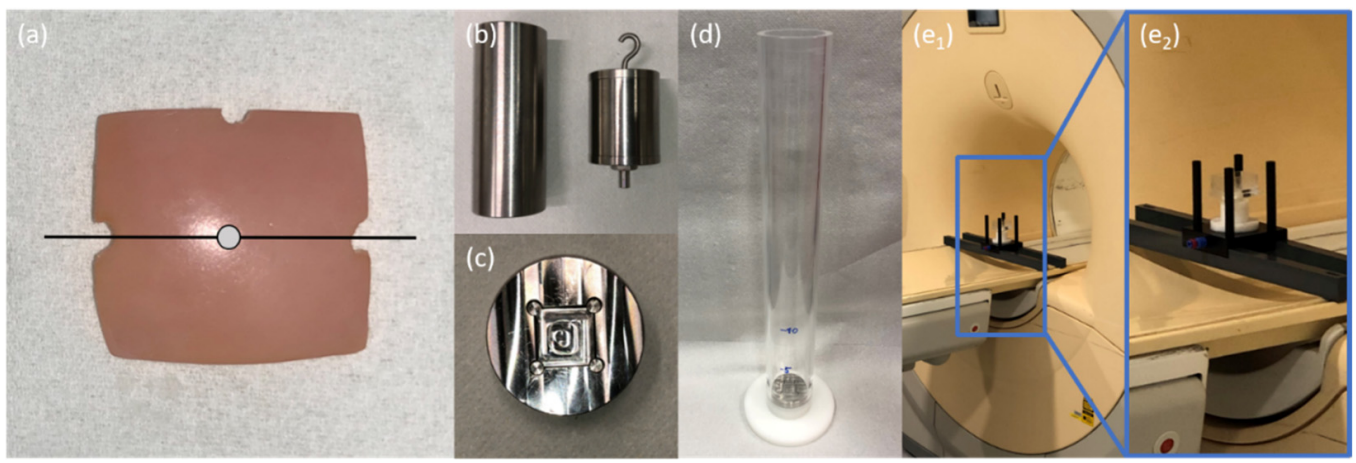

Figure 1. Details of cartilage sample preparation and subsequent injurious impaction loading. (a) Top view of a representative osteochondral sample. Notches at the 3 and 9 o'clock positions indicate the mid-sagittal plane (black line), while the sample centre point is defined as the intersection of this midsagittal plane and its perpendicular along the notch at the 12 o'clock position (grey dot). (b) Weights of $1000 \mathrm{~g}$ (left) and $500 \mathrm{~g}$ (right) equipped with hook and $5 \mathrm{~mm}$-tip to induce standardized injurious impaction. (c) Metallic sample plate with flat recess to fix cartilage samples during impaction by preventing lateral displacement. Seeming distortions are secondary to the milling process and room lights. (d) Cylindrical drop-tower device with metallic sample plate and indications of height. Impaction energy levels were regulated by adjustment of weight and height. Details of the assembled measurement framework at variable magnifications (blue boxes) within the scanner's bore. The framework included support beams mounted on the MRI table $\left(\mathbf{e}_{\mathbf{1}}\right)$ and a transparent sample box (containing the cartilage samples, $\left(\mathbf{e}_{2}\right)$ ) for reproducible positioning of sample and coil (not shown).

Before the study, minimum sample size had been projected using a dedicated online tool (https: / / www.statstodo.com, accessed 3 February 2018). Based on comparable studies $[25,27]$ and assuming a statistical power of 0.9 , a type-I error probability of 0.05 , a maximum inter-group difference of 0.6 , and an intra-group standard deviation of 0.5 , we determined a minimum sample size of 28 (Cohen's effect size model f2). Finally, 35 samples were thus prepared and transferred to 12-well plates filled with DMEM and additives as above.

\subsection{Cartilage Sample Impaction}

Following their preparation, cartilage samples were allocated to three groups, i.e., unimpacted controls (CONT, $\mathrm{n}=7$ ), low impact (LIMP, $0.49 \mathrm{~J}, \mathrm{n}=14$ ), and high impact (HIMP, $0.98 \mathrm{~J}, \mathrm{n}=14$ ). A custom-made drop tower device was used to standardize impaction energy levels as before [32] (Figure 1b-d). Briefly, its specifications (height $33 \mathrm{~cm}$; diameter $4 \mathrm{~cm}$ ) allowed dropping standard cylindric iron weights (500 g or $1000 \mathrm{~g}$, equipped with a 5-mm-diameter tip) from defined heights. In this study, these weights were dropped 
from a height of $100 \mathrm{~mm}$, thereby exposing the cartilage samples to two impaction energy levels of $0.49 \mathrm{~J}$ and $0.98 \mathrm{~J}$ (Table 1) [32,41]. Velocity of impact (v) was determined by drop height (h) (Equation (1)) and energy I by mass (m) and height (h) (Equation (2)), where $g$ is gravity-induced acceleration.

$$
\begin{gathered}
\mathrm{V}=(2 g \mathrm{~h})^{1 / 2} \\
\mathrm{E}=\mathrm{m} g \mathrm{~h}
\end{gathered}
$$

Table 1. Details as a function of impaction characteristics.

\begin{tabular}{ccccc}
\hline Group & Mass $(\mathbf{g})$ & Height $(\mathbf{m m})$ & Velocity $(\mathbf{m} / \mathbf{s})$ & Energy $(\mathbf{J})$ \\
\hline Low Impact (LIMP) & 500 & 100 & 1.4 & 0.49 \\
High Impact (HIMP) & 1000 & 100 & 1.4 & 0.98 \\
\hline
\end{tabular}

These two different impaction energy levels created "mild" (LIMP) and "severe" (HIMP) structural and compositional damage in cartilage [32,41]. To ensure localized impaction at the sample centre point without lateral displacement, a custom-made metallic sample plate with a dedicated recess (depth: $2 \mathrm{~mm}$, width $\times$ height: $16 \mathrm{~mm}$ ) confined and fixed the cartilage samples on all sides (Figure 1c). After impaction, the weight's tip was left to rest on the samples for $5 \mathrm{~s}$ to ensure constant compression conditions [42].

\subsection{MRI Measurements}

Pre- and post-impaction MRI measurements were performed on a clinical 3.0 T MRI scanner (Achieva, Philips, Best, The Netherlands) within $6 \mathrm{~h}$ after sample preparation. Serial MRI measurements were performed just before $\left(\mathrm{t}_{0}\right)$ and immediately after impaction $\left(t_{1}\right)$ as well as $24 \mathrm{~h}\left(t_{2}\right)$ and $72 \mathrm{~h}\left(\mathrm{t}_{3}\right)$ after impaction (Figure 2$)$.

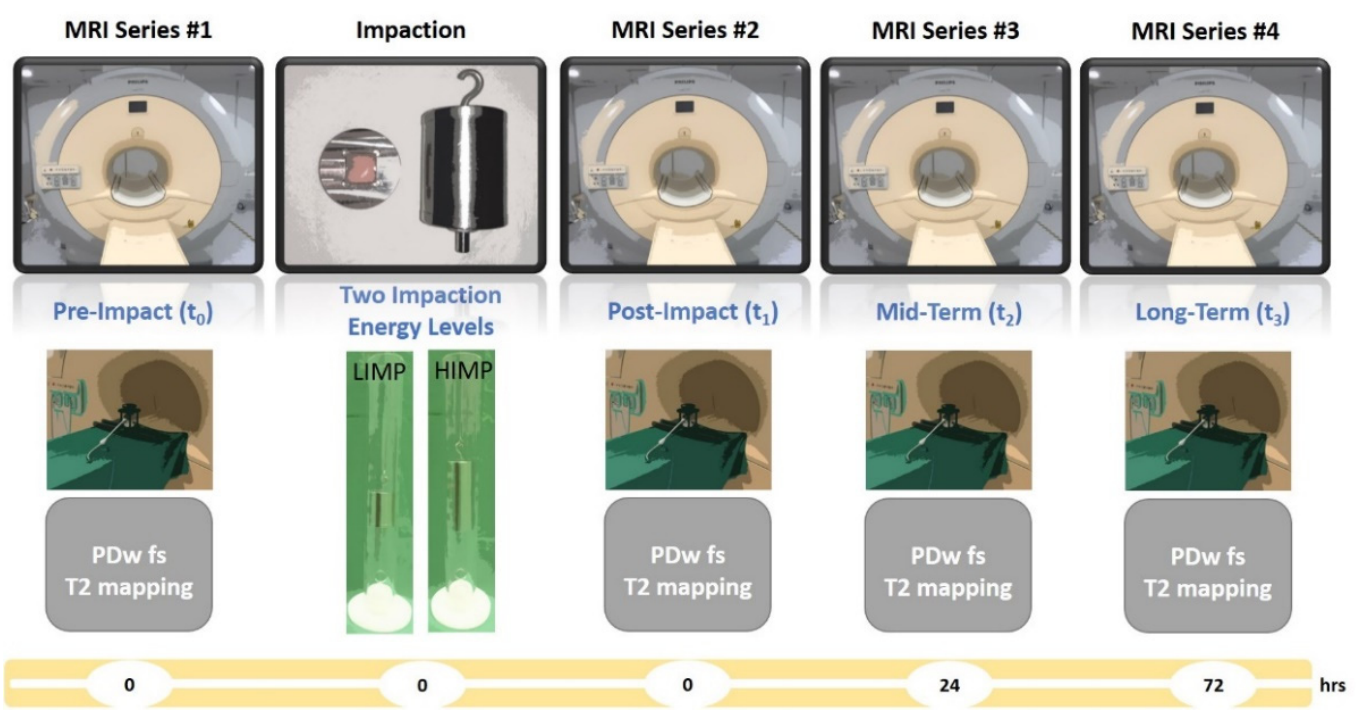

Figure 2. MRI series timeline. Cartilage samples were imaged immediately before $\left(t_{0}\right)$ and after impaction $\left(t_{1}\right)$ as well as after another $24 \mathrm{~h}\left(t_{2}\right)$ and $72 \mathrm{~h}\left(t_{3}\right)$. The imaging protocol was completed for each MRI series (grey boxes). Unit of timeline (yellow) is hours.

For reproducible positioning of cartilage samples in the MRI scanner, a validated MRIcompatible device $[39,43]$ was used. The device's lower frame contained the transparent sample box with the cartilage samples and was mounted on the patient table by means of dedicated support beams (Figure 1e). Thus, imaging was performed close to the scanner's iso-centre with the sample surfaces and mid-sagittal planes parallel to the main magnetic field $\mathrm{B}_{0}$. A modified single-channel receive-only prostate coil (BPX-40 disposable endorectal coil, Medrad/Bayer, Germany) without the inflatable balloon tip was used for imaging and circumferentially enclosed the sample box. Radiofrequency pulses were applied via 
the scanner's in-built body coil. Two cartilage samples were imaged at a time using morphologic and quantitative MRI (Table 2). Briefly, following scout views, Proton Densityweighted sequences were acquired in the sagittal, coronal, and axial orientations. These sequences were used to guide the T2 mapping sequence along the mid-sagittal plane as indicated by the notches at opposite sample sides. Notably, off-the-shelve fish oil capsules (1000 mg) were attached to the sample box for identification purposes. Total imaging time per MRI series was $11 \mathrm{~min} 40 \mathrm{sec}$ and total imaging time per sample pair was $46 \mathrm{~min} 40 \mathrm{~s}$. Measurements were carried out at room temperature, which was monitored during one representative MRI series $\left(20.3 \pm 0.5^{\circ} \mathrm{C}\right)$. After the respective MRI series, the cartilage samples were retrieved, placed in DMEM and additives, and cultured under standard conditions $\left(5 \% \mathrm{CO}_{2} ; 37^{\circ} \mathrm{C}\right.$; humid air) in a standard incubation unit. Culture medium was changed after each MRI series.

Table 2. Acquisition parameters of MR sequences.

\begin{tabular}{|c|c|c|}
\hline Parameters & T2 Map & PD-Weighted \\
\hline Sequence type & multi-spin echo & turbo-spin echo \\
\hline Orientation & mid-sag $^{1}$ & ax, cor, sag ${ }^{2}$ \\
\hline Repetition time [ms] & 1500 & $1500-1589$ \\
\hline Echo time $[\mathrm{ms}]$ & $n \times 8.38(n=1-12)$ & 11 \\
\hline Turbo spin echo factor $[\mathrm{n}]$ & 12 & 6 \\
\hline Field of view [mm] & $52 \times 52$ & $62 \times 62$ \\
\hline Acquisition matrix [pixels] & $176 \times 176$ & $144 \times 142$ \\
\hline Reconstruction matrix [pixels] & $224 \times 224$ & $256 \times 256$ \\
\hline Pixel size $[\mathrm{mm} /$ pixel $]$ & $0.23 \times 0.23$ & $0.24 \times 0.24$ \\
\hline Flip angle $\left[{ }^{\circ}\right]$ & 90 & 90 \\
\hline Number of signal averages [n] & 2 & 2 \\
\hline Slices $[\mathrm{n}]$ & 1 & $8-24$ \\
\hline Slice thickness [mm] & 2.0 & 1.0 \\
\hline Slice gap [mm] & - & 0.5 \\
\hline Duration [min sec] & $4 \min 29 s$ & $7 \min 11 \mathrm{~s}^{3}$ \\
\hline
\end{tabular}

${ }^{1}$ mid-sag-mid-sagittal, ${ }^{2}$ ax-axial, cor-coronal, sag-sagittal, ${ }^{3}$ total duration of all three sequences.

\subsection{MRI Data Analysis}

The T2 maps were generated in a pixel-wise manner using customized mono-exponential fitting routines implemented in MATLAB (MatlabR2020a, Natick, MA, USA) as before [35,39]. For fitting, only echo times $2-7$ (i.e., echo times $<60 \mathrm{~ms}$ with the exception of the first echo to avoid artefacts secondary to stimulated echoes) were included because of the insufficiently low signal-to-noise ratios beyond $60 \mathrm{~ms}$. Cartilage samples were segmented manually by delineating the sample outlines on a moderately T2-weighted morphologic image $(\mathrm{TE}=41.9 \mathrm{~ms})$. To prevent partial volume effects and subsequent $\mathrm{T} 2$ quantification errors, segmentation outlines were delineated conservatively, i.e., only pixels that certainly constituted cartilage tissue were included. Besides the entire sample's cross-section, additional regions-of-interest (ROIs) were defined by automatically dividing the samples into the superficial and deep cartilage layers. Based on another customized MATLAB routine and the segmentation outlines, sample height was determined along the sample's entire width to automatically create two equally thick layers. M.S.H. (2 years of experience in musculoskeletal imaging) performed the manual segmentations that were quality checked by S.N. (9 years of experience in musculoskeletal imaging). For each ROI, summary statistics, i.e., means \pm standard deviations, and texture variables, i.e., variance, contrast, homogeneity, and energy, were calculated using customized MATLAB routines based on earlier approaches $[44,45]$. Information on the spatial arrangement of T2 values were derived using gray-level co-occurrence matrices (GLCMs) for each texture feature. GLCMs serve to tabulate the frequency with which pixel value combinations are present and may be used to determine numerous texture features. Separate GLCMs were determined for each texture variable with an offset of a single pixel based on vertical, horizontal, and angular 
orientations, i.e., $0^{\circ}, 45^{\circ}, 90^{\circ}$, and $135^{\circ}$ [45]. Of note, orientation-dependent inputs were averaged [25]. Metrics of contrast, i.e., contrast and homogeneity, metrics of orderliness, i.e., energy, and statistical metrics, i.e., variance, were calculated [17]:

1. Contrast assesses the extent of local variation. Cartilage areas with high contrast values display strong contrasts, i.e., pronounced differences between the highest and lowest $\mathrm{T} 2$ values.

2. Homogeneity serves as a measure of uniformity by indicating similarities between pixels and their neighbours. Cartilage areas with mostly similar T2 values have high homogeneity values.

3. Energy as computed based on the GLCM provides a measure of uniformity and orderliness. Cartilage areas with high energy values display similar T2 values and small $\mathrm{T} 2$ value differences in neighbouring pixels.

4. Variance is a measure of local variation around the mean. High variance values indicate high heterogeneity and large differences in $\mathrm{T} 2$ values, i.e., variation from their mean.

\subsection{Histologic Reference Analysis}

Following the last MRI series at $t_{3}$, the cartilage samples and the adjacent cartilage tissue underwent standard histological work-up [32,46]. Samples and adjacent tissue were simultaneously decalcified and fixed in Ossa fixona (Diagonal, Muenster, Germany), sectioned along the mid-sagittal plane (or parallel to it), embedded in paraffin, cut to 5- $\mu \mathrm{m}$ sections, and stained with haematoxylin/eosin and Safranin O. Histologic evaluation, including documentation, was performed on a standard light microscope (Leica DM/LM-P, Wetzlar, Germany).

Baseline cartilage status of the adjacent cartilage tissue was assessed semi-quantitatively according to the Mankin classification of cartilage degeneration [47]. Two investigators, i.e., M.S.H. and S.N. with 3 and 11 years of experience in musculoskeletal histopathology, individually assessed each tissue specimen. The Mankin classification assesses tissue structure (score, 0-6), cellularity (score, 0-3), proteoglycan staining intensity (score, $0-4$ ), and tidemark integrity (score, $0-1$ ). Based on each tissue feature's individual score, the Mankin sum score (MSS) gives the cumulative score of degeneration. Ranging from 0-14, lower or higher MSSs indicate less or more severe signs of histologic degeneration, respectively. In case of differing scores, respective histologic sections were discussed until consensus was reached. Of note, only grossly intact cartilage samples with MSS of 0-4 were included.

Following exposure to impaction or control conditions, cartilage samples were assessed semi-quantitatively based on the Mankin classification. Posttraumatic cartilage changes such as alterations in tissue structure, i.e., clefts and other signs of surface disintegration, as well as composition, i.e., Safranin O de-staining, were noted.

\subsection{Statistical Analysis}

Statistical analysis was performed by MSH and SN using GraphPad Prism Software (Version 8.0.2, San Diego, CA, USA). Not assuming normal distributions, absolute T2 values and texture features were compared as a function of time (i.e., between $t_{0}, t_{1}, t_{2}$, and $t_{3}$ ) using Friedman's test followed by Dunn's post hoc test. Accordingly, relative changes for each measure $\left(\Delta_{\mathrm{x}}\right)$ at $t_{\mathrm{x}}$ were determined in reference to $t_{0}$ and calculated using (Equation (3)):

$$
\Delta_{\mathrm{x}}=\left(\left(\mathrm{T} 2 \mathrm{t}_{\mathrm{x}} / \mathrm{T} 2 \mathrm{t}_{0}\right)-1\right) \times 100[\%]
$$

The different impaction energy levels and control conditions at $t_{x}$ were comparatively evaluated using the Kruskal-Wallis test. Group-wise, one-way ANOVA followed by Tukey's post hoc tests (wherever appropriate) were used to compare relative changes $\Delta_{\mathrm{X}}$ as well as histological changes of cartilage structure and proteoglycan content. Results are displayed as mean \pm standard deviation. Due to this study's exploratory character and the large number of comparisons involved, the level of significance was set to $p \leq 0.01$ to 
reduce the number of statistically significant, yet clinically and scientifically (most likely) insignificant findings.

\section{Results}

All 35 samples underwent full MRI and histologic reference evaluation.

\subsection{Macroscopic Reference Evaluation}

Macroscopic evaluation revealed no fracturing of the subchondral bone lamella in any sample. However, considerable dents corresponding to the metallic tip's geometry were observed at the impaction site following HIMP exposure (11/14 samples) (Figure 3a), while no such marks were observed after LIMP exposure or in controls.
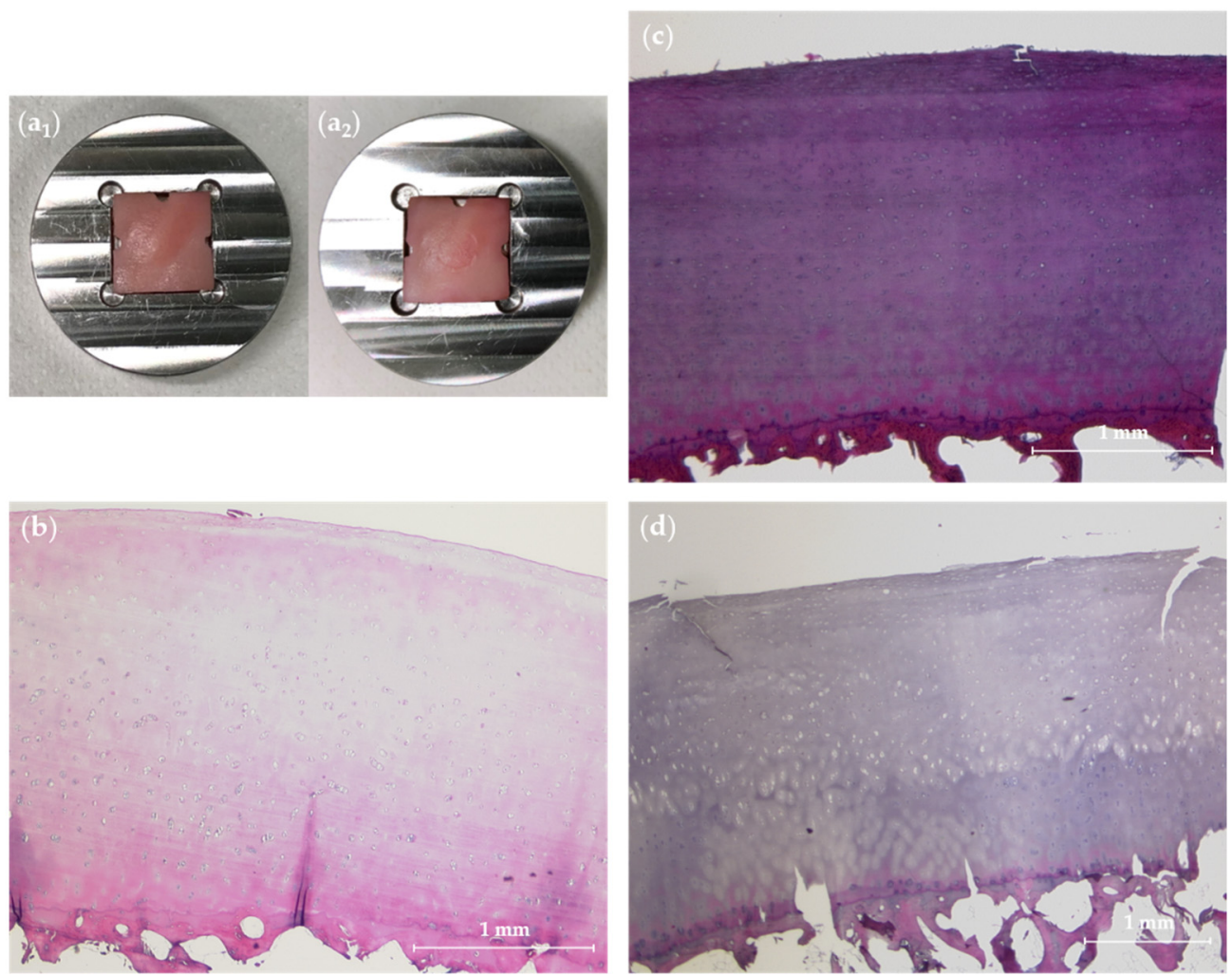

Figure 3. Representative macroscopic and histologic findings after injurious impaction of cartilage samples. (a) Representative cartilage sample positioned in metallic sample plate before ( $\left.\mathbf{a}_{\mathbf{1}}\right)$ and after $\left(\mathbf{a}_{2}\right)$ impaction at high energy (HIMP). Visual inspection revealed clearly visible dent at the area of impaction. (b) Histologically grossly intact adjacent cartilage tissue with slight hypercellularity (Mankin sum score 2), but otherwise normal, indicates gross cartilage integrity at baseline. (c) Cartilage sample after exposure to low-energy impaction (LIMP) with a single cleft at the tissue surface, yet without any other histologic signs of posttraumatic cartilage degeneration. (d) Cartilage sample after HIMP exposure with distinct surface clefts marking the area of impaction and adjacent surface irregularities. Haematoxylin/eosin staining. Scale bars indicate $1 \mathrm{~mm}$.

\subsection{Histologic Reference Evaluation}

Histologic assessment revealed that adjacent cartilage tissue was grossly intact as indicated by mean MSSs of $1.8 \pm 0.8$ (range, $0-3$ ). Baseline cartilage status was dominated by slight signs of histologic degeneration such as surface fibrillation, focal hypercellularity or slight Safranin-O de-staining, translating to MSSs of 1 or 2 in most tissue specimens.

Cartilage samples harvested after impaction and/or standard incubation revealed impaction energy level-associated tissue damage. Even though not significant, surface disintegration was higher with higher impaction energy levels (Table 3). Similarly, nonsignificant differences were observed for Safranin O de-staining that tended to be more 
severe after HIMP than LIMP exposure. Qualitatively, Figure 3b-d gives representative histologic findings.

Table 3. Histologic changes in cartilage samples $72 \mathrm{~h}$ after impaction (LIMP, HIMP) or incubation under control conditions. Based on the Mankin classification [47], surface integrity (score 0-6) and proteoglycan staining intensity (score 0-4) scores are indicated as absolute changes relative to baseline. Group-wise differences were assessed using one-way ANOVA tests. CONT—controls, LIMP—low impaction energy, HIMP—high impaction energy.

\begin{tabular}{ccccc}
\hline Histologic Cartilage Change & CONT & LIMP & HIMP & $p$-Value \\
\hline Surface Integrity & $0.14 \pm 0.34$ & $0.43 \pm 0.82$ & $1.14 \pm 1.4$ & 0.100 \\
Proteoglycan Staining Intensity & $0.14 \pm 1.25$ & $0.64 \pm 1.17$ & $0.93 \pm 1.03$ & 0.163 \\
\hline
\end{tabular}

\subsection{MRI Data—Descriptive Statistics}

For T2, global and zonal changes were found as a function of time and impaction energy level (Table 4).

Table 4. Mean absolute T2 values as a function of region-of-interest, time point, and impaction energy level. T2 values are given as means \pm standard deviation [ms] for the entire cartilage sample as well as the respective superficial and deep tissue layers. Longitudinal (time-related) differences in T2 values were assessed by the Friedman test and respective p-values are organized in columns and indicated by (§). Cross-sectional (group-related) differences in T2 values at individual time points were assessed using the Kruskal-Wallis test and respective $p$-values are organized in lines and indicated by ( $\ddagger)$. Post hoc test details are given in Supplementary Table S1. CONT—controls, LIMP—low-energy impaction, HIMP—high-energy impaction. Significant differences are indicated in bold type.

\begin{tabular}{|c|c|c|c|c|c|c|}
\hline \multirow{2}{*}{ Region-of-Interest } & \multirow{2}{*}{ Group } & \multicolumn{4}{|c|}{ Time } & \multirow{2}{*}{$p$-Value (§) } \\
\hline & & $\mathbf{t}_{\mathbf{0}}$ & $t_{1}$ & $t_{2}$ & $t_{3}$ & \\
\hline \multirow{4}{*}{ Entire Cartilage Sample } & CONT & $33.3 \pm 5.1$ & $34.4 \pm 6.1$ & $35.5 \pm 6.2$ & $35.1 \pm 7.4$ & 0.093 \\
\hline & LIMP & $32.0 \pm 2.4$ & $33.8 \pm 3.6$ & $38.7 \pm 4.5$ & $40.3 \pm 5.2$ & $<0.001$ \\
\hline & HIMP & $35.5 \pm 5.1$ & $40.8 \pm 5.9$ & $45.4 \pm 8.3$ & $55.9 \pm 8.3$ & $<0.001$ \\
\hline & $p$-value $(\ddagger)$ & 0.234 & 0.016 & 0.019 & 0.002 & \\
\hline \multirow{4}{*}{ Superficial Layer } & CONT & $37.0 \pm 6.4$ & $39.1 \pm 7.6$ & $39.5 \pm 7.2$ & $39.0 \pm 8.8$ & 0.180 \\
\hline & LIMP & $38.2 \pm 4.3$ & $42.4 \pm 5.2$ & $46.3 \pm 4.4$ & $47.5 \pm 6.1$ & $<0.001$ \\
\hline & HIMP & $42.9 \pm 5.8$ & $48.8 \pm 6.3$ & $54.5 \pm 9.7$ & $67.2 \pm 19.7$ & $<0.001$ \\
\hline & $p$-value ( $\ddagger)$ & 0.026 & 0.008 & 0.001 & 0.001 & \\
\hline \multirow{4}{*}{ Deep Layer } & CONT & $29.4 \pm 5.1$ & $29.4 \pm 5.1$ & $31.4 \pm 6.2$ & $31.1 \pm 6.6$ & 0.18 \\
\hline & LIMP & $26.1 \pm 2.8$ & $28.1 \pm 3.8$ & $32.8 \pm 7.7$ & $33.9 \pm 6.9$ & $<0.001$ \\
\hline & HIMP & $27.7 \pm 6.0$ & $32.6 \pm 7.1$ & $36.2 \pm 9.2$ & $44.4 \pm 11.4$ & $<0.001$ \\
\hline & $p$-value ( $)$ & 0.247 & 0.008 & 0.557 & 0.011 & \\
\hline
\end{tabular}

In controls, T2 values remained largely constant even though, by trend, a slight and non-significant progressive increase was noted for all ROIs, from $33.3 \pm 5.1 \mathrm{~ms}\left(\mathrm{t}_{0}\right)$ to $35.1 \pm 7.4 \mathrm{~ms}\left(\mathrm{t}_{3}\right)\left(p=0.093\right.$, entire cartilage sample), from $37.0 \pm 6.4 \mathrm{~ms}\left(\mathrm{t}_{0}\right)$ to $39.0 \pm 8.8 \mathrm{~ms}$ $\left(\mathrm{t}_{3}\right)\left(p=0.180\right.$, superficial layer), and from $29.4 \pm 5.1 \mathrm{~ms}\left(\mathrm{t}_{0}\right)$ to $31.1 \pm 6.6 \mathrm{~ms}\left(\mathrm{t}_{3}\right)(p=0.180$, deep layer). After LIMP exposure, T2 values were significantly increased in all ROIs, from $32.0 \pm 2.4 \mathrm{~ms}\left(\mathrm{t}_{0}\right)$ to $40.3 \pm 5.2 \mathrm{~ms}\left(\mathrm{t}_{3}\right)(p<0.001$, entire cartilage sample), from $38.2 \pm 4.3 \mathrm{~ms}\left(\mathrm{t}_{0}\right)$ to $47.5 \pm 6.1 \mathrm{~ms}\left(\mathrm{t}_{3}\right)(p<0.001$, superficial layer), and from $26.1 \pm 2.8 \mathrm{~ms}$ $\left(\mathrm{t}_{0}\right)$ to $33.9 \pm 6.9 \mathrm{~ms}\left(\mathrm{t}_{3}\right)(p<0.001$, deep layer). Post hoc analysis revealed these differences to be significant between $t_{0}$ and $t_{2}$ and between $t_{0}$ and $t_{3}$ (Supplementary Table S1). Similarly, after HIMP exposure, T2 values were significantly increased in all ROIs, too, from $35.5 \pm 5.1 \mathrm{~ms}\left(\mathrm{t}_{0}\right)$ to $55.9 \pm 8.3 \mathrm{~ms}\left(\mathrm{t}_{3}\right)(p<0.001$, entire cartilage sample), from 
$42.9 \pm 5.8 \mathrm{~ms}\left(\mathrm{t}_{0}\right)$ to $67.2 \pm 19.7 \mathrm{~ms}\left(\mathrm{t}_{3}\right)(p<0.001$, superficial layer $)$, and from $27.7 \pm 6.0 \mathrm{~ms}$ $\left(\mathrm{t}_{0}\right)$ to $44.4 \pm 11.4 \mathrm{~ms}\left(\mathrm{t}_{3}\right)(p<0.001$, deep layer). Significant post hoc differences were found between $t_{0}$ and $t_{1}$ (except for deep layer), $t_{0}$ and $t_{2}$, and $t_{0}$ and $t_{3}$ (Supplementary Table S1).

At individual time points, group-wise, i.e., impaction energy-related, differences in T2 were significant primarily in the superficial layers at $t_{1}, t_{2}$, and $t_{3}$ (Table 4 ). Post hoc analysis revealed the differences to be significant when comparing controls and HIMPexposed samples, indicating significantly higher T2 values after HIMP exposure than in controls (Supplementary Table S2). Relative changes of T2 values confirmed these findings (Table 5). When considering the entire cartilage sample, the magnitude of relative changes gradually increased as a function of time, i.e., $\Delta_{1}<\Delta_{3}$, and impaction energy level, i.e., CONT $<$ LIMP $<$ HIMP. Statistical analysis revealed these changes to be significant for $\Delta_{1}$ and $\Delta_{3}$ only, while $\Delta_{2}$ tended towards significance in all ROIs. Post hoc analysis indicated that the differences were significant only for the CONT vs. HIMP comparison, while CONT vs. LIMP or LIMP vs. HIMP were not significant (Supplementary Table S3).

Table 5. Relative changes of $\mathrm{T} 2$ values as a function of region-of-interest, time point, and impaction energy level. Relative changes $(\Delta)$ were calculated as $\Delta_{\mathrm{x}}=\left(\left(\mathrm{T} 2 \mathrm{t}_{\mathrm{x}} / \mathrm{T} 2 \mathrm{t}_{0}\right)-1\right) \times 100[\%]$ for the time points $t_{1}, t_{2}$, and $t_{3}$. For each time point, $\Delta$ values were compared in a group-wise manner based on one-way ANOVA tests. Significant differences are indicated in bold type. For an explanation of the abbreviations please refer to Table 4 .

\begin{tabular}{ccccc}
\hline Region of Interest & Group & $\boldsymbol{\Delta}_{\mathbf{1}}$ & $\boldsymbol{\Delta}_{\mathbf{2}}$ & $\boldsymbol{\Delta}_{\mathbf{3}}$ \\
\hline \multirow{3}{*}{ Entire Cartilage Sample } & CONT & $3.0 \pm 3.4$ & $6.7 \pm 5.4$ & $4.7 \pm 6.5$ \\
\cline { 2 - 5 } & LIMP & $9.5 \pm 3.5$ & $23.7 \pm 17.6$ & $27.1 \pm 16.4$ \\
\cline { 2 - 5 } & HIMP & $15.3 \pm 8.3$ & $28.6 \pm 16.1$ & $59.2 \pm 42.1$ \\
\cline { 2 - 5 } & $\boldsymbol{p}$-value & $<\mathbf{0 . 0 0 1}$ & 0.012 & $<\mathbf{0 . 0 0 1}$ \\
\cline { 2 - 5 } Superficial Layer & CONT & $5.5 \pm 5.1$ & $6.8 \pm 6.3$ & $4.9 \pm 8.8$ \\
\cline { 2 - 5 } & LIMP & $10.9 \pm 11.0$ & $22.5 \pm 23.9$ & $25.1 \pm 27.2$ \\
\cline { 2 - 5 } & HIMP & $14.0 \pm 14.2$ & $27.6 \pm 29.3$ & $57.1 \pm 61.5$ \\
\hline \multirow{2}{*}{ Deep Layer } & $\boldsymbol{p}$-value & $\mathbf{0 . 0 0 2}$ & 0.041 & $\mathbf{0 . 0 0 3}$ \\
\cline { 2 - 5 } & CONT & $0.4 \pm 5.9$ & $6.7 \pm 9.0$ & $-1.2 \pm 5.1$ \\
\cline { 2 - 5 } & LIMP & $7.8 \pm 7.7$ & $25.4 \pm 27.0$ & $29.9 \pm 31.4$ \\
\cline { 2 - 5 } & HIMP & $18.4 \pm 18.0$ & $30.9 \pm 32.3$ & $63.6 \pm 67.1$ \\
\hline & $\boldsymbol{p}$-value & 0.013 & 0.019 & $<\mathbf{0 . 0 0 1}$ \\
\hline
\end{tabular}

\subsection{MRI Data-Texture Feature Analysis}

Radiomic texture features were significantly different only after HIMP exposure, and not in controls or after LIMP exposure (Table 6). Metrics of contrast displayed distinct changes. Contrast values were undulating in controls, while after LIMP exposure, samples' contrast values tended to increase from $0.25 \pm 0.17\left(t_{0}\right)$ to $0.28 \pm 0.11\left(t_{3}\right)(p=0.270)$. After HIMP exposure, however, contrast values were significantly increased from $0.24 \pm 0.09$ $\left(t_{0}\right)$ to $0.40 \pm 0.17\left(t_{3}\right)(p<0.001)$. Opposite observations were made for homogeneity that remained relatively constant in controls $(p=0.615)$ and tended to decrease in LIMP-exposed samples $(p=0.166)$ but decreased significantly from $0.88 \pm 0.05\left(t_{0}\right)$ to $0.82 \pm 0.06\left(t_{3}\right)$ $(p<0.001)$ in HIMP-exposed samples. Energy as a metric of orderliness was characterized by distinct changes, too. While energy was undulating in controls $(p=0.510)$, it decreased considerably from $0.43 \pm 0.20\left(\mathrm{t}_{0}\right)$ to $0.30 \pm 0.07\left(\mathrm{t}_{3}\right)(p=0.016)$ and from $0.35 \pm 0.13\left(\mathrm{t}_{0}\right)$ to $0.24 \pm 0.16\left(\mathrm{t}_{3}\right)(p=0.006)$ after LIMP and HIMP exposure, respectively, thereby tending towards (LIMP) or reaching statistical significance (HIMP). Variance as a statistical metric was undulating, too, in controls, while it underwent moderate, yet non-significant increases 
from $111.0 \pm 94.9\left(\mathrm{t}_{0}\right)$ to $143.2 \pm 69.4\left(\mathrm{t}_{3}\right)(p=0.015)$ following LIMP exposure and strong and significant increases from $118.3 \pm 79.9\left(t_{0}\right)$ to $311.4 \pm 264.8\left(t_{3}\right)(p=0.005)$ following HIMP exposure. Group-wise comparisons at individual time points revealed no significant differences at $t_{0}$, indicating largely similar baseline texture feature and sample homogeneity prior to exposure. For contrast, homogeneity, and energy, significant group-wise differences were found at $t_{2}$ and $t_{3}$, while for variance, significant differences were only observed at $t_{3}$.

Table 6. Absolute values of radiomic texture features as a function of impaction energy level and time point. Values are given as means \pm standard deviation for the entire cartilage samples. Please see Table 4 for details on table organization, statistical analysis, and abbreviations. P-values of time-related differences in T2 values (Friedman test) are organized in columns (§) and p-values of group-related differences in T2 values (Kruskal-Wallis test) are organized in lines ( $\ddagger$ ). Significant differences are indicated in bold type.

\begin{tabular}{|c|c|c|c|c|c|c|c|}
\hline \multirow{2}{*}{ Texture Feature Class } & \multirow{2}{*}{ Texture Feature } & \multirow{2}{*}{ Groups } & \multicolumn{4}{|c|}{ Time } & \multirow[t]{2}{*}{$p$-Value $(\S)$} \\
\hline & & & $t_{0}$ & $t_{1}$ & $t_{2}$ & $t_{3}$ & \\
\hline \multirow{8}{*}{ Metrics of Contrast } & \multirow{4}{*}{ Contrast } & CONT & $0.18 \pm 0.07$ & $0.19 \pm 0.06$ & $0.18 \pm 0.04$ & $0.17 \pm 0.06$ & 0.615 \\
\hline & & LIMP & $0.25 \pm 0.17$ & $0.27 \pm 0.14$ & $0.26 \pm 0.08$ & $0.28 \pm 0.11$ & 0.270 \\
\hline & & HIMP & $0.24 \pm 0.09$ & $0.30 \pm 0.09$ & $0.30 \pm 0.09$ & $0.40 \pm 0.17$ & $<0.001$ \\
\hline & & $p$-value ( $)$ & 0.312 & 0.06 & 0.003 & 0.001 & \\
\hline & \multirow{4}{*}{ Homogeneity } & CONT & $0.91 \pm 0.03$ & $0.91 \pm 0.02$ & $0.91 \pm 0.02$ & $0.91 \pm 0.03$ & 0.615 \\
\hline & & LIMP & $0.89 \pm 0.05$ & $0.87 \pm 0.05$ & $0.87 \pm 0.03$ & $0.87 \pm 0.04$ & 0.166 \\
\hline & & HIMP & $0.88 \pm 0.05$ & $0.86 \pm 0.04$ & $0.86 \pm 0.03$ & $0.82 \pm 0.06$ & 0.001 \\
\hline & & $p>$-value ( $)$ & 0.256 & 0.074 & 0.003 & 0.001 & \\
\hline \multirow{4}{*}{ Metric of Orderliness } & \multirow{4}{*}{ Energy } & CONT & $0.49 \pm 0.19$ & $0.46 \pm 0.17$ & $0.46 \pm 0.11$ & $0.48 \pm 0.15$ & 0.510 \\
\hline & & LIMP & $0.43 \pm 0.20$ & $0.36 \pm 0.16$ & $0.32 \pm 0.07$ & $0.30 \pm 0.07$ & 0.016 \\
\hline & & HIMP & $0.35 \pm 0.13$ & $0.30 \pm 0.10$ & $0.28 \pm 0.10$ & $0.24 \pm 0.16$ & 0.006 \\
\hline & & $p$-value ( $)$ & 0.225 & 0.078 & 0.004 & 0.002 & \\
\hline \multirow{4}{*}{ Statistical Metric } & \multirow{4}{*}{ Variance } & CONT & $69.5 \pm 28.7$ & $77.4 \pm 33.0$ & $70.5 \pm 27.1$ & $67.1 \pm 29.4$ & 0.392 \\
\hline & & LIMP & $111.0 \pm 94.9$ & $142.1 \pm 92.6$ & $128.2 \pm 58.6$ & $143.2 \pm 69.4$ & 0.015 \\
\hline & & HIMP & $118.3 \pm 79.9$ & $157.3 \pm 101.6$ & $189.7 \pm 124.6$ & $311.4 \pm 264.8$ & 0.005 \\
\hline & & $p$-value ( $\ddagger)$ & 0.277 & 0.118 & 0.014 & 0.010 & \\
\hline
\end{tabular}

\subsection{MRI Data-Image Evaluation}

Impaction-induced quantitative changes as outlined above were reflected by corresponding qualitative changes in the T2 maps (Figure 4). Controls remained largely unchanged and maintained the inherent depth-wise stratification of T2 values with lower values in deeper and higher values in the more superficial cartilage zones. After LIMP exposure, the typical depth-wise stratification was progressively lost and gradually replaced by a band-like hyperintense signal zone at the sample centre. The hyperintense zone was not confined to the area of impaction, extended throughout the entire sample width, and was oriented parallel to the subchondral lamella. After HIMP exposure, the depth-wise stratification was lost immediately and superseded by a more diffuse and widespread area of hyperintense signal that gradually increased in size and hyperintensity to eventually involve the large parts of the sample's cross-sectional area. Once settled, this area remained largely constant in the mid-to-long term. Supplementary Figure S1 gives more representative cartilage samples and their post-impaction changes. 


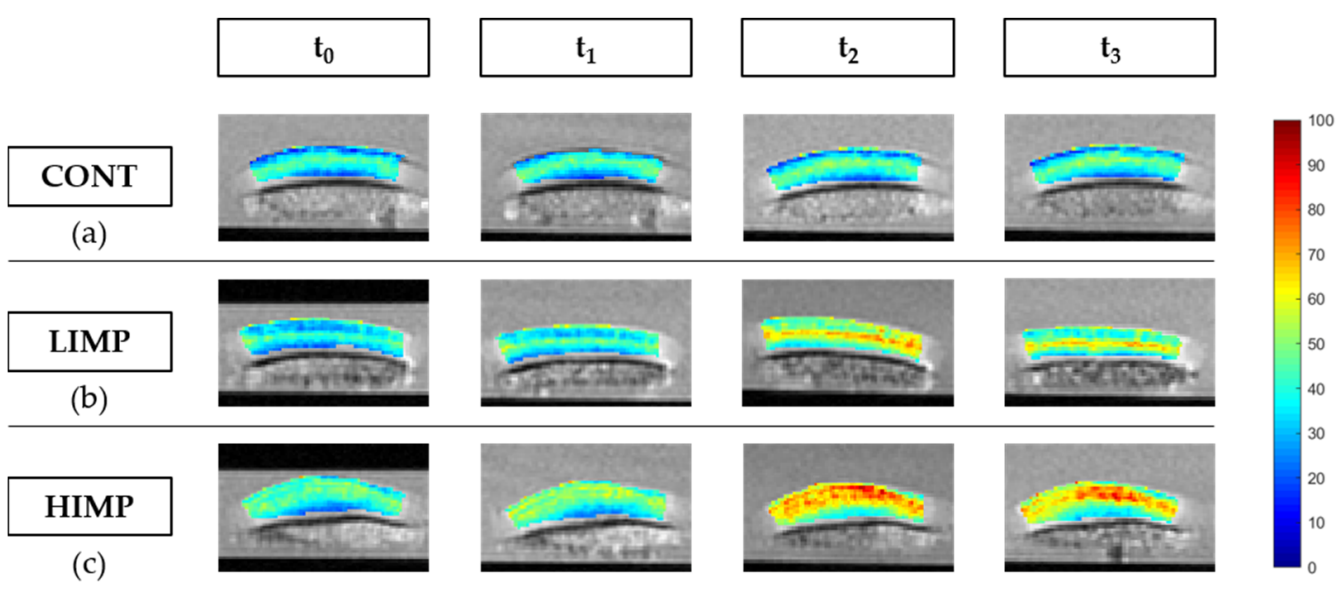

Figure 4. Color-coded and spatially resolved T2 parameter maps of representative cartilage samples and their post-impaction changes. Control samples without impaction (a). Cartilage samples displayed distinct changes after low-energy impaction (b) and more pronounced and widespread changes after high-energy impaction (c). Time points are marked by $t_{0}, t_{1}, t_{2}$, and $t_{3}$ that indicate the pre- $\left(t_{0}\right)$ and post-impaction measurements $\left(t_{1}\right)$ as well as those after $24 h\left(t_{2}\right)$ and $72 h\left(t_{3}\right)$. Scale bar on the right indicates color-coded T2 values [ms].

\section{Discussion}

The most important finding of this study is that advanced MRI acquisition and postprocessing techniques, i.e., quantitative $\mathrm{T} 2$ mapping and texture feature analysis, may be used to (i) differentiate the severity of supraphysiological impact injuries of cartilage and (ii) monitor post-traumatic degenerative changes.

Prior to its initiation, this study had been motivated by the lack of basic research available on the association of T2 mapping and traumatic cartilage injury. Even though a solid body of clinical evidence is available to support the potential of T2 mapping in evaluating posttraumatic cartilage changes [17], the changes in T2 related to traumatic injury are variable and inconsistent. In young adults with recurrent patellar dislocations during childhood, significant decreases in T2 were found in the superficial patellar cartilage zone of injured as compared to non-injured joints, which may be an early sign of cartilage pathology [18]. After ACL reconstruction, inconsistent T2 changes relative to uninjured controls were found. At the one-year follow-up, T2 was not significantly elevated in one study [19], while others found significant T2 elevations at the medial femoral cartilage after two [20] and three years [21]. In contrast, increases in T2 were associated with morphologic cartilage lesions [22] as well as morphologically intact cartilage that is going to develop morphologic cartilage lesions in the years to come [23]. This, of course, indicates that compositional changes -as assessed by T2 mapping- precede the development of morphologic cartilage lesions and underscores the potential of T2 mapping to identify cartilage regions at risk of incipient degeneration.

This study clearly demonstrates that injurious cartilage impaction is associated with increasing T2 values as a function of impaction energy level and time. Based on the sensitivity profile of $\mathrm{T} 2$ versus structural and compositional cartilage properties, these increases reflect numerous single-impact-associated posttraumatic changes in cartilage $[32,41,42,48-51]$. The literature data indicate that these changes include surface damage, loss of proteoglycans and collagen network integrity, as well as chondrocyte death. These changes closely resemble degenerative changes in OA, are therefore often referred to as traumatic OA-like changes [42], and provide the degenerative correlates of altered $\mathrm{T} 2$ values. For the sake of comparability, impaction energy levels were chosen in line with earlier studies and, mechanistically, the induction of cartilage damage by dropping weights from defined heights has been thoroughly validated before $[32,41,48]$. Nonetheless, in this study, histologic reference indicated impaction-energy-associated surface disintegration 
and incipient-to-moderate proteoglycan depletion, thereby confirming the mode of action within the framework of this study.

On the tissue level, the impaction-induced increases in $\mathbf{T} 2$ may be secondary to numerous posttraumatic changes that are excellently reviewed in [52]. For once, proteoglycans (and -in parts- collagen) are lost secondary to the collagen network damage. Lower proteoglycan and collagen contents are associated with higher T2 values [53]. For another, collagen network disintegration is induced directly by mechanical disruption and indirectly by subsequent enzymatic degradation. These processes contribute to increased tissue water content and tissue swelling, which are associated with higher T2 values, too [33], as well as increased collagen fibre disorientation and anisotropy. Secondary to impaction, the percentage of fibres oriented at magic angle, i.e., at $55^{\circ}$ to the main magnetic field, may be elevated, thereby increasing T2 values, too [54]. Yet, even though these mechanisms are plausible, it remains unclear which exact compositional or (ultra)structural mechanism is primarily behind the prominent increases in $\mathrm{T} 2$.

Beyond mean T2 values, this study focused on radiomic texture features, too, as refined imaging biomarkers of cartilage trauma. Again, changes were clear and significant after HIMP exposure, while they were moderate and only tended towards significance after LIMP exposure. Across the spectrum of texture features assessed, variance and metrics of contrast and orderliness were significantly increased (i.e., contrast, variance) and decreased (i.e., homogeneity, energy) as a function of impaction energy. Additionally, these features were different between the various time points, indicating lower textural uniformity and growing structural disorder. These changes may be considered a sign of cartilage damage, too, as described before $[25,26,44]$.

Notably, changes in T2, i.e., absolute values and texture features, were subject to gradual and drastic changes over time. These aspects may be explained by the concurrence of posttraumatic changes in cartilage that are induced either immediately through the impaction itself or delayed through cell death and the induction of matrix-degrading enzymes. These processes reduce biosynthetic capacity or bring about progressive degradation that may explain the gradual alterations in T2 characteristics. Most likely, these processes are at the root of the hyperintense bands that traversed the cartilage sample in the transitional zone parallel to the subchondral lamella. Observed after both low- and highenergy exposure, similar histologic changes have been reported before [32,41]. Following impaction of bovine cartilage, Jeffrey et al. found horizontal fissures in the transitional zone that they hypothesized to be due to deflection of the extracellular matrix with partial delamination of the upper and lower tissue portions. Other studies also demonstrated sub-surface intra-tissue damage and chondrocyte death prior to surface disintegration [55]. The band's progression in terms of size and signal characteristics was clearly associated with impaction energy level and may thus reflect the ongoing structural and compositional changes of traumatized cartilage.

Notably, zonal changes of the superficial and deep tissue layers were roughly similar in terms of relative changes in T2. Considering the direct impaction of the cartilage surface, intuitively, one would expect larger changes of superficial than deeper zones. Yet, the viscoelastic nature of cartilage, its unique compressive properties and tight attachment to the underlying subchondral bone provide efficient mechanisms for absorption of physiologic and supraphysiologic loads throughout the entire tissue depth [56]. Once the subchondral bone is removed, these mechanisms of load distribution and dissipation are disrupted and the protective effect is lost [41].

This study has numerous limitations. First, the experimental in vitro design necessitated excision and preparation of cartilage and its prolonged incubation, thereby limiting the clinical translatability of our findings. Prolonged incubation in media may artificially increase tissue hydration, thereby increasing $\mathrm{T} 2$ values. As this was observed for otherwise unaffected control samples, these gradual increases in T2 provide the background against which the impaction-induced changes must be considered. Additionally, scanning was performed at room temperature, again affecting T2 values. As the MRI measurements 
were performed in a standardized manner, this bias may be considered systematic. Additional studies using in situ human whole-knee joint configurations and more physiologic impaction methods are thus required to confirm our findings. As an additional caveat, however, resultant intra-tissue changes secondary to impaction are largely dependent on the experimental framework conditions. Standardized impaction of cartilage samples induces more severe changes than similar impaction of intact joints [57]. Second, human cartilage samples were obtained from knee joints undergoing joint replacement. Despite our best efforts to ascertain tissue quality by macroscopic evaluation and baseline histology, the pre-existent degeneration of the tissue brought about by chronic mechanical and inflammatory disease processes is clearly uncontrolled and may have altered the tissue's susceptibility to impaction loading. Even though the longitudinal study design allows for consistent intra-sample referencing and reduces this type of bias, tissue variability clearly affects tissue susceptibility and outcomes of impaction. Future studies should therefore use 'truly' healthy cartilage through alternative tissue sources such as amputations or organdonor networks to realize improved tissue quality control. Third, despite selecting cartilage from the lateral femoral condyles for reasons of topoanatomic consistency, human cartilage thickness is largely different between individuals [58] and may affect load distribution and dissipation in the tissue. Fourth, the cartilage samples surfaces were oriented parallel to the main magnetic field B0 and, consequently, the majority of collagen fibres of the deep and transitional zones were oriented at $90^{\circ}$ to $\mathrm{B} 0$, thereby affecting $\mathrm{T} 2$ quantification. T2 values may increase due to the magic angle effect as collagen fibre orientation changes relative to $B_{0}$ after injurious impaction, particularly in the deep tissue layer [54] Although measurement conditions were standardized (using a dedicated device) and inter-sample variability in position and configuration thus decreased, this aspect needs to be considered, too, prior to any clinical translation. Fifth, this study only focused on T2 mapping, while other imaging markers, e.g., T1 $\rho, \mathrm{T} 1, \mathrm{~T} 2$ *, sodium, gagCEST (glycosaminoglycan Chemical Exchange Saturation Transfer), or contrast-enhanced techniques such as dGEMRIC (delayed gadolinium-enhanced MRI of cartilage) are of potential value in assessing cartilage structure and composition [14,34,38,59-64]. Of these, T1 $\rho$ mapping is of additional and complementary value to T2 mapping because of distinct biophysical properties [65]. Yet, the exact sensitivity profile of T1 $\rho$ remains to be determined with proteoglycan, collagen, and water content as well as collagen fibre orientation potentially contributing to T1 $\rho$ relaxation characteristics. As of today, T1 $\rho$ seems to indicate the cartilage tissue' macromolecular configuration [53,66-69]. In posttraumatic contexts, the sensitivity of T1 $\rho$ to changes in the tissue's solid and fluid constituents and its mechanical condition [38,70-73] may be of value in future pre-clinical and clinical studies. Sixth, this study did not include dedicated compositional reference measures and, consequently, no advanced quantification of proteoglycan or collagen content that would have allowed spatially resolved associations of T2 maps and compositional measures. Prior to any clinical translation, these associations ought to be clarified in posttraumatic contexts and beyond.

\section{Conclusions}

This study demonstrates that advanced MRI acquisition and postprocessing techniques, i.e., quantitative T2 mapping and texture feature analysis, are sensitive diagnostic means to detect and monitor traumatic impaction injuries of cartilage and associated posttraumatic degenerative changes. If corroborated by additional in situ and in vivo studies, the close association of changes in T2 and texture features, impaction energy level, and time render this technique diagnostically promising to assess cartilage tissue after trauma and to detect cartilage at risk. 
Supplementary Materials: The following are available online at https:/ / www.mdpi.com/2075-172 9/11/3/201/s1, Figure S1: Additional examples of color-coded and spatially resolved T2 parameter maps of representative cartilage samples and their post-impaction changes. Table S1: Post hoc details of absolute T2 values as a function of time-point-wise comparisons, Table S2: Post hoc details of absolute T2 values as a function of group-wise comparisons, Table S3: Post hoc comparisons of relative changes.

Author Contributions: Conceptualization, M.S.H. and S.N.; methodology, M.S.H., D.T. and S.N.; software, M.S.H., D.T., J.S., K.L.R., and S.N.; validation, M.S.H., M.P., D.T., C.K., and S.N.; formal analysis, M.S.H., J.S., K.L.R., and S.N.; investigation, M.S.H. and S.N.; resources, M.S.H., D.B.A., M.P., D.T., C.K., and S.N.; data curation, M.S.H., J.S., K.L.R. and S.N.; writing-original draft preparation, M.S.H.; writing-review and editing, M.S.H. and S.N.; visualization, M.S.H. and S.N.; supervision, C.K., D.T. and S.N.; project administration, S.N.; funding acquisition, S.N. All authors have read and agreed to the published version of the manuscript.

Funding: This research was funded by (i) the Deutsche Forschungsgemeinschaft (grant number: NE 2136/3-1), (ii) the Research Commission of the Medical Faculty of Heinrich-Heine-University Düsseldorf, and (iii) the START Program of the Faculty of Medicine, RWTH Aachen, Germany.

Institutional Review Board Statement: The study was conducted according to the guidelines of the Declaration of Helsinki, and approved by the Institutional Ethical Review Board of the Medical Faculty of RWTH Aachen University (AZ EK 157/13).

Informed Consent Statement: Informed consent was obtained from all patients whose cartilage-bone material was included in the study.

Data Availability Statement: The raw data presented in this study are available from the corresponding author upon reasonable request.

Acknowledgments: We gratefully acknowledge Sophie Lecouturier for her comprehensive support and the Aachen Interdisciplinary Center for Clinical Research for the provision of histologic processing facilities and equipment.

Conflicts of Interest: The authors declare no conflict of interest. The funders had no role in the design of the study; in the collection, analyses, or interpretation of data; in the writing of the manuscript, or in the decision to publish the results.

\section{References}

1. Hjelle, K.; Solheim, E.; Strand, T.; Muri, R.; Brittberg, M. Articular cartilage defects in 1000 knee arthroscopies. Arthroscopy 2002, 18, 730-734. [CrossRef] [PubMed]

2. Widuchowski, W.; Lukasik, P.; Kwiatkowski, G.; Faltus, R.; Szyluk, K.; Widuchowski, J.; Koczy, B. Isolated full thickness chondral injuries. Prevalance and outcome of treatment. A retrospective study of 5233 knee arthroscopies. Acta Chir. Orthop. Traumatol. Cech. 2008, 75, 382-386. [PubMed]

3. Widuchowski, W.; Widuchowski, J.; Trzaska, T. Articular cartilage defects: Study of 25,124 knee arthroscopies. Knee 2007, 14, 177-182. [CrossRef]

4. Curl, W.W.; Krome, J.; Gordon, E.S.; Rushing, J.; Smith, B.P.; Poehling, G.G. Cartilage injuries: A review of 31,516 knee arthroscopies. Arthroscopy 1997, 13, 456-460. [CrossRef]

5. Brown, T.D.; Johnston, R.C.; Saltzman, C.L.; Marsh, J.L.; Buckwalter, J.A. Posttraumatic osteoarthritis: A first estimate of incidence, prevalence, and burden of disease. J. Orthop. Trauma 2006, 20, 739-744. [CrossRef]

6. Vollnberg, B.; Koehlitz, T.; Jung, T.; Scheffler, S.; Hoburg, A.; Khandker, D.; Hamm, B.; Wiener, E.; Diederichs, G. Prevalence of cartilage lesions and early osteoarthritis in patients with patellar dislocation. Eur. Radiol. 2012, 22, 2347-2356. [CrossRef] [PubMed]

7. Thomas, A.C.; Hubbard-Turner, T.; Wikstrom, E.A.; Palmieri-Smith, R.M. Epidemiology of posttraumatic osteoarthritis. J. Athl. Train. 2017, 52, 491-496. [CrossRef]

8. De Maeseneer, M.; Shahabpour, M.; Pouders, C. MRI spectrum of medial collateral ligament injuries and pitfalls in diagnosis. JBR-BTR 2010, 93, 97-103.

9. Halinen, J.; Koivikko, M.; Lindahl, J.; Hirvensalo, E. The efficacy of magnetic resonance imaging in acute multi-ligament injuries. Int. Orthop. 2009, 33, 1733. [CrossRef]

10. Farshad-Amacker, N.A.; Potter, H.G. MRI of knee ligament injury and reconstruction. J. Magn. Reson. Imaging 2013, 38, 757-773. [CrossRef]

11. Von Engelhardt, L.V.; Kraft, C.N.; Pennekamp, P.H.; Schild, H.H.; Schmitz, A.; von Falkenhausen, M. The evaluation of articular cartilage lesions of the knee with a 3-Tesla magnet. Arthrosc. J. Arthrosc. Relat. Surg. 2007, 23, 496-502. [CrossRef] 
12. Figueroa, D.; Calvo, R.; Vaisman, A.; Carrasco, M.A.; Moraga, C.; Delgado, I. Knee chondral lesions: Incidence and correlation between arthroscopic and magnetic resonance findings. Arthrosc. J. Arthrosc. Relat. Surg. 2007, 23, 312-315. [CrossRef] [PubMed]

13. Kajabi, A.W.; Casula, V.; Ojanen, S.; Finnila, M.A.; Herzog, W.; Saarakkala, S.; Korhonen, R.K.; Nissi, M.J.; Nieminen, M.T. Multiparametric MR imaging reveals early cartilage degeneration at 2 and 8 weeks after ACL transection in a rabbit model. J. Orthop. Res. 2020, 38, 1974-1986. [CrossRef]

14. Nebelung, S.; Post, M.; Knobe, M.; Tingart, M.; Emans, P.; Thuring, J.; Kuhl, C.; Truhn, D. Detection of early-stage degeneration in human articular cartilage by multiparametric mr imaging mapping of tissue functionality. Sci. Rep. 2019, 9, 5895. [CrossRef] [PubMed]

15. Kijowski, R.; Blankenbaker, D.G.; Munoz Del Rio, A.; Baer, G.S.; Graf, B.K. Evaluation of the articular cartilage of the knee joint: Value of adding a T2 mapping sequence to a routine MR imaging protocol. Radiology 2013, 267, 503-513. [CrossRef]

16. Neu, C.P. Functional imaging in OA: Role of imaging in the evaluation of tissue biomechanics. Osteoarthr. Cartil. 2014, 22, 1349-1359. [CrossRef] [PubMed]

17. Eagle, S.; Potter, H.G.; Koff, M.F. Morphologic and quantitative magnetic resonance imaging of knee articular cartilage for the assessment of post-traumatic osteoarthritis. J. Orthop. Res. 2017, 35, 412-423. [CrossRef]

18. Bengtsson Mostrom, E.; Lammentausta, E.; Finnbogason, T.; Weidenhielm, L.; Janarv, P.M.; Tiderius, C.J. Pre- and postcontrast T1 and T2 mapping of patellar cartilage in young adults with recurrent patellar dislocation. Magn. Res. Med. 2015, 74, 1363-1369. [CrossRef]

19. Li, X.; Kuo, D.; Theologis, A.; Carballido-Gamio, J.; Stehling, C.; Link, T.M.; Ma, C.B.; Majumdar, S. Cartilage in anterior cruciate ligament-reconstructed knees: MR imaging T1 $\rho$ and T2-initial experience with 1-year follow-up. Radiology 2011, 258, 505-514. [CrossRef] [PubMed]

20. Su, F.; Hilton, J.F.; Nardo, L.; Wu, S.; Liang, F.; Link, T.M.; Ma, C.B.; Li, X. Cartilage morphology and T1 $\rho$ and T2 quantification in ACL-reconstructed knees: A 2-year follow-up. Osteoarthr. Cartil. 2013, 21, 1058-1067. [CrossRef] [PubMed]

21. Bae, J.-H.; Hosseini, A.; Wang, Y.; Torriani, M.; Gill, T.J.; Grodzinsky, A.J.; Li, G. Articular cartilage of the knee 3 years after ACL reconstruction: A quantitative T2 relaxometry analysis of 10 knees. Acta Orthop. 2015, 86, 605-610. [CrossRef]

22. Årøen, A.; Brøgger, H.; Røtterud, J.H.; Sivertsen, E.A.; Engebretsen, L.; Risberg, M.A. Evaluation of focal cartilage lesions of the knee using MRI T2 mapping and delayed Gadolinium Enhanced MRI of Cartilage (dGEMRIC). BMC Musculoskelet. Disord. 2016, 17, 73. [CrossRef]

23. Kretzschmar, M.; Nevitt, M.C.; Schwaiger, B.J.; Joseph, G.B.; McCulloch, C.E.; Link, T.M. Spatial distribution and temporal progression of $\mathrm{T} 2$ relaxation time values in knee cartilage prior to the onset of cartilage lesions-Data from the Osteoarthritis Initiative (OAI). Osteoarthr. Cartil. 2019, 27, 737-745. [CrossRef] [PubMed]

24. Saarakkala, S.; Julkunen, P.; Kiviranta, P.; Makitalo, J.; Jurvelin, J.S.; Korhonen, R.K. Depth-wise progression of osteoarthritis in human articular cartilage: Investigation of composition, structure and biomechanics. Osteoarthr. Cartil. 2010, 18, 73-81. [CrossRef]

25. Joseph, G.B.; Baum, T.; Carballido-Gamio, J.; Nardo, L.; Virayavanich, W.; Alizai, H.; Lynch, J.A.; McCulloch, C.E.; Majumdar, S.; Link, T.M. Texture analysis of cartilage T2 maps: Individuals with risk factors for OA have higher and more heterogeneous knee cartilage MR T2 compared to normal controls-Data from the osteoarthritis initiative. Arthr. Res. Ther. 2011, 13, R153. [CrossRef]

26. Joseph, G.; Baum, T.; Alizai, H.; Carballido-Gamio, J.; Nardo, L.; Virayavanich, W.; Lynch, J.; Nevitt, M.; McCulloch, C.; Majumdar, $\mathrm{S}$. Baseline mean and heterogeneity of MR cartilage T2 are associated with morphologic degeneration of cartilage, meniscus, and bone marrow over 3 years-Data from the Osteoarthritis Initiative. Osteoarthr. Cartil. 2012, 20, 727-735. [CrossRef]

27. Peuna, A.; Hekkala, J.; Haapea, M.; Podlipská, J.; Guermazi, A.; Saarakkala, S.; Nieminen, M.T.; Lammentausta, E. Variable angle gray level co-occurrence matrix analysis of $\mathrm{T} 2$ relaxation time maps reveals degenerative changes of cartilage in knee osteoarthritis: Oulu knee osteoarthritis study. J. Magne. Reson. Imaging 2018, 47, 1316-1327. [CrossRef]

28. Baum, T.; Joseph, G.B.; Nardo, L.; Virayavanich, W.; Arulanandan, A.; Alizai, H.; Carballido-Gamio, J.; Nevitt, M.C.; Lynch, J.; McCulloch, C.E. Correlation of magnetic resonance imaging-based knee cartilage T2 measurements and focal knee lesions with body mass index: Thirty-six-month followup data from a longitudinal, observational multicenter study. Arthr. Care Res. 2013, 65, 23-33. [CrossRef] [PubMed]

29. Williams, A.; Winalski, C.S.; Chu, C.R. Early articular cartilage MRI T2 changes after anterior cruciate ligament reconstruction correlate with later changes in T2 and cartilage thickness. J. Orthop. Res. 2017, 35, 699-706. [CrossRef] [PubMed]

30. Repo, R.U.; Finlay, J.B. Survival of articular cartilage after controlled impact. J. Bone Joint Surg. Am. 1977, 59, 1068-1076. [CrossRef]

31. Torzilli, P.A.; Grigiene, R.; Borrelli, J., Jr.; Helfet, D.L. Effect of impact load on articular cartilage: Cell metabolism and viability, and matrix water content. J. Biomech. Eng. 1999, 121, 433-441. [CrossRef] [PubMed]

32. De Bont, F.; Brill, N.; Schmitt, R.; Tingart, M.; Rath, B.; Pufe, T.; Jahr, H.; Nebelung, S. Evaluation of single-impact-induced cartilage degeneration by optical coherence tomography. BioMed Res. Int. 2015, 2015, 486794. [CrossRef] [PubMed]

33. Linka, K.; Itskov, M.; Truhn, D.; Nebelung, S.; Thuring, J. T2 MR imaging vs. computational modeling of human articular cartilage tissue functionality. J. Mech. Behav. Biomed. Mater. 2017, 74, 477-487. [CrossRef]

34. Nebelung, S.; Brill, N.; Tingart, M.; Pufe, T.; Kuhl, C.; Jahr, H.; Truhn, D. Quantitative OCT and MRI biomarkers for the differentiation of cartilage degeneration. Skelet. Radiol. 2016, 45, 505-516. [CrossRef]

35. Nebelung, S.; Post, M.; Knobe, M.; Shah, D.; Schleich, C.; Hitpass, L.; Kuhl, C.; Thuring, J.; Truhn, D. Human articular cartilage mechanosensitivity is related to histological degeneration-A functional MRI study. Osteoarthr. Cartil. 2019, 27, 1711-1720. [CrossRef] 
36. Nebelung, S.; Post, M.; Raith, S.; Fischer, H.; Knobe, M.; Braun, B.; Prescher, A.; Tingart, M.; Thuring, J.; Bruners, P.; et al. Functional in situ assessment of human articular cartilage using MRI: A whole-knee joint loading device. Biomech. Model. Mechanobiol. 2017, 16, 1971-1986. [CrossRef]

37. Nebelung, S.; Sondern, B.; Jahr, H.; Tingart, M.; Knobe, M.; Thuring, J.; Kuhl, C.; Truhn, D. Non-invasive T1rho mapping of the human cartilage response to loading and unloading. Osteoarthr. Cartil. 2018, 26, 236-244. [CrossRef]

38. Nebelung, S.; Sondern, B.; Oehrl, S.; Tingart, M.; Rath, B.; Pufe, T.; Raith, S.; Fischer, H.; Kuhl, C.; Jahr, H.; et al. Functional MR imaging mapping of human articular cartilage response to loading. Radiology 2017, 282, 464-474. [CrossRef] [PubMed]

39. Hafner, T.; Schock, J.; Post, M.; Abrar, D.B.; Sewerin, P.; Linka, K.; Knobe, M.; Kuhl, C.; Truhn, D.; Nebelung, S. A serial multiparametric quantitative magnetic resonance imaging study to assess proteoglycan depletion of human articular cartilage and its effects on functionality. Sci. Rep. 2020, 10, 15106. [CrossRef] [PubMed]

40. Outerbridge, R.E. The etiology of chondromalacia patellae. J. Bone Joint Surg. Br. 1961, 43, 752-757. [CrossRef]

41. Jeffrey, J.E.; Gregory, D.W.; Aspden, R.M. Matrix damage and chondrocyte viability following a single impact load on articular cartilage. Arch. Biochem. Biophys. 1995, 322, 87-96. [CrossRef]

42. Huser, C.A.; Davies, M.E. Validation of an in vitro single-impact load model of the initiation of osteoarthritis-like changes in articular cartilage. J. Orthop. Res. 2006, 24, 725-732. [CrossRef]

43. Truhn, D.; Brill, N.; Braun, B.; Merhof, D.; Kuhl, C.; Knobe, M.; Thuring, J.; Nebelung, S. A multi-purpose force-controlled loading device for cartilage and meniscus functionality assessment using advanced MRI techniques. J. Mech. Behav. Biomed. Mater. 2020, 101, 103428. [CrossRef]

44. Wilson, K.J.; Surowiec, R.K.; Ho, C.P.; Devitt, B.M.; Fripp, J.; Smith, W.S.; Spiegl, U.J.; Dornan, G.J.; Laprade, R.F. Quantifiable imaging biomarkers for evaluation of the posterior cruciate ligament using 3-T magnetic resonance imaging. Orthop. J. Sports Med. 2016, 4, 232596711663904. [CrossRef]

45. Haralick, R.M.; Shanmugam, K.; Dinstein, I. Textural features for image classification. IEEE Trans. Syst. Man Cybern. 1973, 3, 610-621. [CrossRef]

46. Nebelung, S.; Marx, U.; Brill, N.; Arbab, D.; Quack, V.; Jahr, H.; Tingart, M.; Zhou, B.; Stoffel, M.; Schmitt, R.; et al. Morphometric grading of osteoarthritis by optical coherence tomography-An ex vivo study. J. Orthop. Res. 2014, 32, 1381-1388. [CrossRef]

47. Mankin, H.J.; Dorfman, H.; Lippiello, L.; Zarins, A. Biochemical and metabolic abnormalities in articular cartilage from osteoarthritic human hips. II. Correlation of morphology with biochemical and metabolic data. J. Bone Joint Surg. Am. 1971, 53, 523-537. [CrossRef]

48. Verteramo, A.; Seedhom, B.B. Effect of a single impact loading on the structure and mechanical properties of articular cartilage. J. Biomech. 2007, 40, 3580-3589. [CrossRef]

49. Pritzker, K.P.; Gay, S.; Jimenez, S.A.; Ostergaard, K.; Pelletier, J.P.; Revell, P.A.; Salter, D.; van den Berg, W.B. Osteoarthritis cartilage histopathology: Grading and staging. Osteoarthr. Cartil. 2006, 14, 13-29. [CrossRef]

50. Van de Loo, A.A.; Arntz, O.J.; Otterness, I.G.; van den Berg, W.B. Proteoglycan loss and subsequent replenishment in articular cartilage after a mild arthritic insult by IL-1 in mice: Impaired proteoglycan turnover in the recovery phase. Ag. Act. 1994, 41 , 200-208.

51. Hamerman, D.; Klagsbrun, M. Osteoarthritis. Emerging evidence for cell interactions in the breakdown and remodeling of cartilage. Am. J. Med. 1985, 78, 495-499. [CrossRef]

52. Kurz, B.; Lemke, A.K.; Fay, J.; Pufe, T.; Grodzinsky, A.J.; Schünke, M. Pathomechanisms of cartilage destruction by mechanical injury. Ann. Anat. Anat. Anz. 2005, 187, 473-485. [CrossRef] [PubMed]

53. Menezes, N.M.; Gray, M.L.; Hartke, J.R.; Burstein, D. T2 and T1rho MRI in articular cartilage systems. Magn. Reson. Med. 2004, 51, 503-509. [CrossRef]

54. Shao, H.; Pauli, C.; Li, S.; Ma, Y.; Tadros, A.S.; Kavanaugh, A.; Chang, E.Y.; Tang, G.; Du, J. Magic angle effect plays a major role in both T1rho and T2 relaxation in articular cartilage. Osteoarthr. Cartil. 2017. [CrossRef]

55. Duda, G.N.; Eilers, M.; Loh, L.; Hoffman, J.E.; Kaab, M.; Schaser, K. Chondrocyte death precedes structural damage in blunt impact trauma. Clin. Orthop. Relat. Res. 2001, 393, 302-309. [CrossRef]

56. Mansour, J. Biomechanics of cartilage. In Kinesiology: The Mechanics and Pathomechanics of Human Movement; Oatis, C.A., Ed.; Lippincott Williams and Wilkins: Baltimore, MD, USA, 2004; pp. 66-79.

57. Borrelli, J., Jr.; Ricci, W.M. Acute effects of cartilage impact. Clin. Orthop. Relat. Res. 2004, 33-39. [CrossRef]

58. Schmitz, R.J.; Harrison, D.; Wang, H.M.; Shultz, S.J. Sagittal-plane knee moment during gait and knee cartilage thickness. J. Athl. Train. 2017, 52, 560-566. [CrossRef]

59. Linka, K.; Thuring, J.; Rieppo, L.; Aydin, R.C.; Cyron, C.J.; Kuhl, C.; Merhof, D.; Truhn, D.; Nebelung, S. Machine learningaugmented and microspectroscopy-informed multiparametric MRI for the non-invasive prediction of articular cartilage composition. Osteoarthr. Cartil. 2021. [CrossRef]

60. MacKay, J.W.; Low, S.B.L.; Smith, T.O.; Toms, A.P.; McCaskie, A.W.; Gilbert, F.J. Systematic review and meta-analysis of the reliability and discriminative validity of cartilage compositional MRI in knee osteoarthritis. Osteoarthr. Cartil. 2018, 26, 1140-1152. [CrossRef]

61. Guermazi, A.; Alizai, H.; Crema, M.D.; Trattnig, S.; Regatte, R.R.; Roemer, F.W. Compositional MRI techniques for evaluation of cartilage degeneration in osteoarthritis. Osteoarthr. Cartil. 2015, 23, 1639-1653. [CrossRef] 
62. Abrar, D.B.; Schleich, C.; Frenken, M.; Vordenbaumen, S.; Richter, J.; Schneider, M.; Ostendorf, B.; Nebelung, S.; Sewerin, P. DGEMRIC in the assessment of pre-morphological cartilage degeneration in rheumatic disease: Rheumatoid arthritis vs. psoriatic arthritis. Diagnostics 2021, 11, 147. [CrossRef]

63. Muller-Lutz, A.; Kamp, B.; Nagel, A.M.; Ljimani, A.; Abrar, D.; Schleich, C.; Wollschlager, L.; Nebelung, S.; Wittsack, H.J. Sodium MRI of human articular cartilage of the wrist: A feasibility study on a clinical 3T MRI scanner. MAGMA 2020. [CrossRef]

64. Abrar, D.B.; Schleich, C.; Radke, K.L.; Frenken, M.; Stabinska, J.; Ljimani, A.; Wittsack, H.J.; Antoch, G.; Bittersohl, B.; Hesper, T.; et al. Detection of early cartilage degeneration in the tibiotalar joint using 3 T gagCEST imaging: A feasibility study. MAGMA 2020. [CrossRef]

65. Wang, L.; Regatte, R.R. T1rho MRI of human musculoskeletal system. J. Magn. Reson. Imaging JMRI 2015, 41, 586-600. [CrossRef]

66. Link, T.M.; Neumann, J.; Li, X. Prestructural cartilage assessment using MRI. J. Magn. Reson. Imaging JMRI 2017, 45, 949-965. [CrossRef]

67. Van Tiel, J.; Kotek, G.; Reijman, M.; Bos, P.K.; Bron, E.E.; Klein, S.; Nasserinejad, K.; van Osch, G.J.; Verhaar, J.A.; Krestin, G.P.; et al. Is T1rho mapping an alternative to delayed gadolinium-enhanced $\mathrm{mr}$ imaging of cartilage in the assessment of sulphated glycosaminoglycan content in human osteoarthritic knees? An in vivo validation study. Radiology 2016, 279, 523-531. [CrossRef]

68. Thuring, J.; Linka, K.; Itskov, M.; Knobe, M.; Hitpass, L.; Kuhl, C.; Truhn, D.; Nebelung, S. Multiparametric MRI and computational modelling in the assessment of human articular cartilage properties: A comprehensive approach. BioMed Res. Int. 2018, 2018, 9460456. [CrossRef]

69. Wong, C.S.; Yan, C.H.; Gong, N.J.; Li, T.; Chan, Q.; Chu, Y.C. Imaging biomarker with T1rho and T2 mappings in osteoarthritisIn vivo human articular cartilage study. Eur. J. Radiol. 2013, 82, 647-650. [CrossRef]

70. Hamada, H.; Nishii, T.; Tamura, S.; Tanaka, H.; Wakayama, T.; Sugano, N. Comparison of load responsiveness of cartilage T1rho and T2 in porcine knee joints: An experimental loading MRI study. Osteoarthr. Cartil. 2015, 23, 1776-1779. [CrossRef]

71. Pastrama, M.I.; Ortiz, A.C.; Zevenbergen, L.; Famaey, N.; Gsell, W.; Neu, C.P.; Himmelreich, U.; Jonkers, I. Combined enzymatic degradation of proteoglycans and collagen significantly alters intratissue strains in articular cartilage during cyclic compression. J. Mech. Behav. Biomed. Mater. 2019, 98, 383-394. [CrossRef]

72. Souza, R.B.; Kumar, D.; Calixto, N.; Singh, J.; Schooler, J.; Subburaj, K.; Li, X.; Link, T.M.; Majumdar, S. Response of knee cartilage T1rho and T2 relaxation times to in vivo mechanical loading in individuals with and without knee osteoarthritis. Osteoarthr. Cartil. 2014, 22, 1367-1376. [CrossRef]

73. Subburaj, K.; Souza, R.B.; Stehling, C.; Wyman, B.T.; Le Graverand-Gastineau, M.P.; Link, T.M.; Li, X.; Majumdar, S. Association of MR relaxation and cartilage deformation in knee osteoarthritis. J. Orthop. Res. 2012, 30, 919-926. [CrossRef] 